\title{
Immanent Justice Reasoning by Spatial Proximity
}

\author{
Mitchell J. Callan \\ Department of Psychology, University of Bath \\ Joshua Moreton \\ Department of Psychology, University of Essex \\ Gethin Hughes \\ Department of Psychology, University of Essex
}

In press, Social Psychological and Personality Science (accepted 12 Nov 2019)

\section{CORRESPONDING AUTHOR:}

Mitchell J. Callan

Department of Psychology, University of Bath

Bath, United Kingdom BA2 7AY

m.j.callan@bath.ac.uk

NOTE:

This work was supported, in part, by studentship ES/J500045/1 from the Economic Social and Research Council. 


\begin{abstract}
Immanent justice reasoning involves causally attributing someone's bad outcome to their prior immoral actions. Building on the idea that causality is mentally linked with spatial proximity, we investigated whether such reasoning might lead participants to spatially bind together immoral actions and bad outcomes. Across 4 experiments $(N=553$ Mechanical Turk workers), participants positioned sentences describing other people's bad (vs. good) outcomes closer in space to previous immoral behaviours. This effect was observed both when the position of the initial action remained in a fixed location and when it "chased" the outcome across the screen. Importantly, we also found that this spatial positioning of immoral actions and bad outcomes is mediated by perceived deservingness of the outcome and is not merely due to perceived similarity of events. These findings suggest that perceived deservingness biases the spatial proximity of representations of others' random bad outcomes and their prior immoral actions.
\end{abstract}

Keywords: immanent justice; metaphorical thinking; spatial proximity; deservingness; perceived similarity 


\section{Immanent Justice Reasoning by Spatial Proximity}

Immanent justice reasoning is the belief that actions bring about deserved outcomes, even when there is no physically plausible means by which they might have done so (Piaget, 1932 Callan, Sutton, Harvey, \& Dawtry, 2014). For instance, inferring that a man's freak accident was caused by him having an extramarital affair is reasoning in immanent justice terms (Callan, Ellard, \& Nicol, 2006). Research suggests that immanent justice reasoning stems, in part, from the need to believe in a just world. According to Lerner's (1980) just-world theory, people need to sustain the functional belief that the world is a just, fair, and nonrandom place where people get what they deserve. From this perspective, reasoning that a random bad outcome was caused by someone's prior immoral actions allows people to maintain the belief that things happen for a reason, and the reason can be found in the target's prior misdeeds-people get what they deserve. Evidence for this account comes from research documenting that perceived deservingness underpins people's immanent justice attributions (e.g., Harvey \& Callan, 2014) and immanent justice reasoning increases when the importance of a just-world to people is heightened (e.g., Callan, Harvey, Dawtry, \& Sutton, 2013).

To date researchers have largely investigated immanent justice reasoning by asking participants to rate the degree to which they believe a given outcome (e.g., a freak accident) was caused by the recipient's prior moral conduct (e.g., stealing). Bridging empirical and theoretical insights from social and cognitive psychology, in the current research we examined whether immanent justice reasoning manifests in how people spatially arrange representations of others' immoral actions closer to representations of their fortuitous bad (vs. good) outcomes. Theory and research on metaphorical thinking suggests that abstract concepts are mentally represented in concrete or physical dimensions such as space and time (Lakoff \& Johnson, 1980; Landau, Meier, \& Keefer, 2010; Thibodeau, Matlock, \& Flusberg, 2019). Of relevance to the current research, abstract judgements of causal strength are often understood in terms of concrete forms of physical closeness (Lakoff \& Johnson, 1980). This mental association between spatial proximity and causality-that is, physical closeness equals causal influence-is established from early repeated encounters with mechanical causal relationships in one's environment (Einhorn \& Hogarth, 1986; Michotte, 1963; Rips, 2011; White, 1988). Indeed, when physical events occur closer together in space, people are more likely to infer a causal link between them (Michotte, 1963). The converse is also true: the distance between two moving objects is perceived as smaller when the movement of the objects are causally linked than when they are not 
(Buehner \& Humphreys, 2010). Thus, the conceptual metaphorical link between spatial proximity and causal strength may result in people arranging immoral actions and fortuitous bad (vs. good) outcomes closer together in space, insofar as doing so reflects their immanent justice reasoning.

\section{Overview of research}

In Experiments 1a and 1b, we asked participants to spatially arrange sentences describing good or bad outcomes (e.g., winning a lottery ticket or being in a freak accident, respectively) either towards or away from descriptions of previous immoral actions (e.g., selling drugs). We hypothesized that participants would position bad outcomes closer to the perpetrators of immoral actions, reflecting their tendency to construe such outcomes in immanent justice terms (i.e., as deserved). Research from Conceptual Metaphor Theory (Lakoff \& Johnson, 1980), however, suggests that, like the metaphorical association between causality and physical closeness noted above, "similarity" and "proximity/closeness" are closely mapped in people's minds (e.g., Breaux \& Feist, 2008; Boot \& Pecher, 2010; Casasanto, 2008; Winter \& Matlock, 2013). Thus, spatial positioning of immoral actions and bad outcomes might reflect conceptual similarity of the events rather immanent justice reasoning per se. Indeed, people tend to arrange similar visual and non-visual stimuli closer together in space (Goldstone, 1994; Hout, Goldinger, \& Ferguson, 2013). Winter and Matlock (2013), for example, found that participants positioned stick characters closer together when they believed the characters held similar (vs. dissimilar) political views. Thus, participants might position morally congruent (vs. incongruent) events closer together in space not because they are inferring causality from deservingness but simply because the events are conceptually similar (e.g., poisoning one's dog and contracting an illness are conceptually similar because both are evaluatively bad).

We empirically addressed this alternative explanation in several ways. In our first two experiments, we crossed a Person factor (i.e., the outcome occurred to the same or different person) with congruency of the outcome. That is, along with congruency, we compared whether bad outcomes befell the same person that had behaved immorally to when the same negative event befell a different person. Although Frank, for example, might deserve a bad outcome because he was immoral, Geoff does not deserve a bad outcome because Frank was immoral. Thus, although the value of the actions and outcomes in both cases are similar, we expected congruency to affect spatial positioning more strongly when the outcome occurred to the person who deserved it (i.e., the same person). Observing such a Person X Congruency interaction would help discount conceptual similarity as an 
explanation for the spatial binding of bad outcomes to bad people: If participants use the number of ways the scenarios are similar as cues to determine spatial positioning, then an additive rather than interactive model would provide a better fit (i.e., we would observe main effects of Person and Congruency but no Person X Congruency interaction). Instead, we predicted that the effect of congruency on spatial positioning would be larger when the outcome occurred to the person who deserved it.

In Experiments 2 and 3, we directly examined whether perceived deservingness of the outcome underpins the spatial binding of fortuitous bad outcomes to immoral actions. In Experiment 2, we examined whether perceived deservingness of a target's outcome mediated the effect of moral congruency of actions and outcomes during the spatial binding task used in Experiment 1. Generalizing to a different task involving the movement of shapes representing moral and immoral actions and bad outcomes, in Experiment 3 we predicted that participants would be more inclined to keep the shapes closer together in space when the bad person was "chasing" a bad (vs. good) outcome, and that this effect would be more strongly associated with perceived deservingness of the outcome than with perceived similarity of the events.

\section{Sampling}

We recruited participants from the U.S.A. through Amazon's Mechanical Turk for all experiments. Except for Experiment 3, Part 2 (see footnote 1), the required sample sizes across experiments were determined ahead of data collection; the final sample sizes were not completely predetermined due to the unpredictable nature of online recruitment (e.g., because of slight over-recruitment and removing participants with duplicate IP addresses). We based our sample sizes on achieving at least $80 \%$ power to detect small-to-medium effects. The data and materials for all experiments are available at osf.io/fb7g5/. We report all measures, manipulations, and exclusions.

\section{Experiments 1a and 1b}

\section{Method}

Participants. We recruited 80 (44 males; $\left.M_{\text {age }}=34.60, S D_{\text {age }}=10.26\right)$ and 62 (30 males, 31 females, 1 preferred not to say: $\left.M_{\text {age }}=34.79, S D_{\text {age }}=11.23\right)$ participants for Experiments $1 \mathrm{a}$ and $1 \mathrm{~b}$, respectively. We excluded an additional 3 participants from Experiment 1a due to duplicate IP addresses (we retained the earliest response).

\section{Materials and procedures.}

Experiment 1a. We developed a measure where participants moved two individual sentences describing two events of a scenario closer together or further apart in space. 
There were 8 positions that the sentences could occupy (see Figure 1). At the beginning of each trial, a sentence describing an immoral action occupied the first position and a sentence describing a good or bad outcome occupied the fifth position. Participants were instructed to, for each trial, click and drag the sentences closer together or further apart according to how physically close or far apart the two events felt to them. Each trial was scored from 1 (the sentences were placed next to each other) to 7 (the sentences were placed as far apart as possible). Prior to the main block, participants completed 1 practice trial with neutral sentences ("David took his bicycle to the corner store" and "Ross bought a new jigsaw puzzle").

Experiment 1b. Experiment $1 \mathrm{~b}$ was the same as Experiment 1a except that the participants could freely move the "outcome" sentence to any position on the screen (cf. Goldstone, 1994). The sentence describing the immoral action always occupied a fixed position in the centre of the screen. Each sentence was fixed to a black circle positioned directly above the middle of the sentence (see Figure 1). For each trial, the starting position of the moveable sentence was below the stationary sentence, two-thirds vertically of the display-screen. We asked participants to position the circles as close or as far apart as they wanted. We calculated the absolute distance between the centre of the screen (the initial misdeed) and the position of the moveable ball (the outcome) and divided this by the maximum possible distance (i.e., from the centre of the screen to the corner of the screen) dependent on each participant's screen. We then multiplied this value by 100 , to give a score representing the percentage of the maximum possible distance the two circles could be separated. 


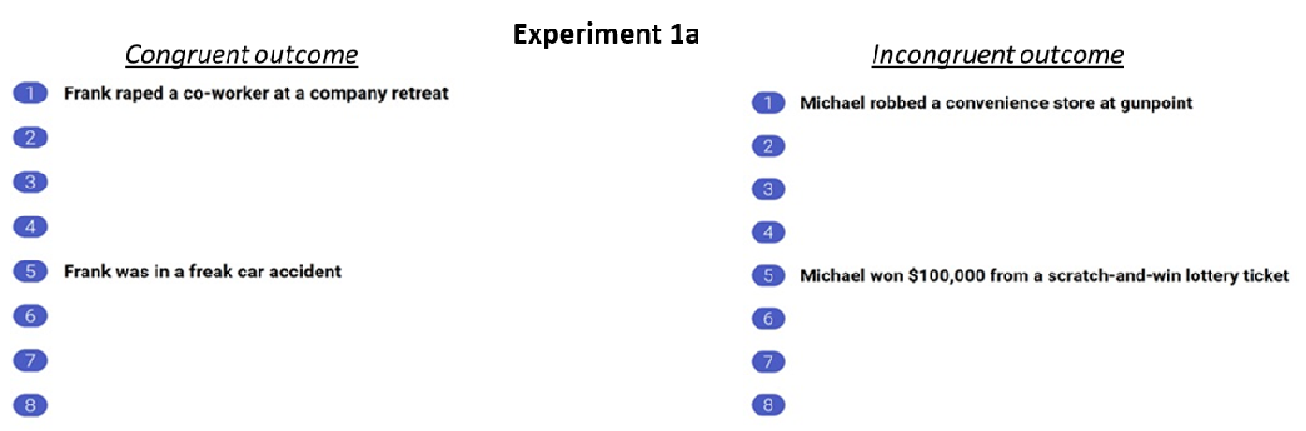

Experiment 1b
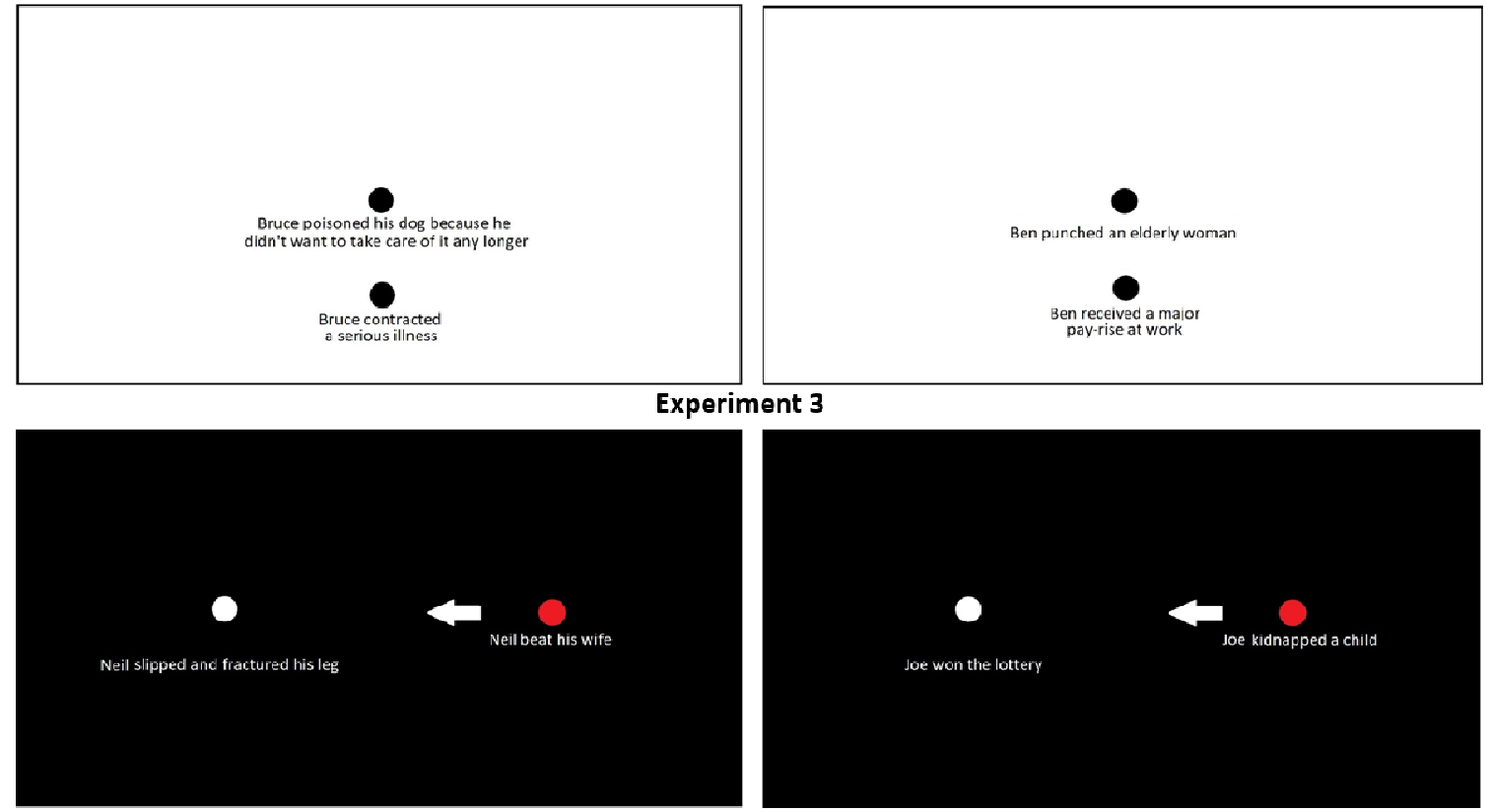

Figure 1. Examples of congruent (left) and incongruent (right) trials/scenarios across experiments. The images depict the starting positions of the immoral actions and outcomes for each trial. For Experiment 3, the white arrow illustrates the starting path of the red circle's motion once the trial began.

Scenarios and design. The scenarios we used for Experiments $1 \mathrm{a}$ and $1 \mathrm{~b}$ represented the conditions of a fully-within 2 (Congruency: morally congruent vs. morally incongruent) $\times 2$ (Person: same vs. different) factorial design. In each scenario, the individual performed an immoral action. Congruency was manipulated by varying the value of the outcome (bad vs. good). For the Person factor, we manipulated whether the outcome occurred to the same or a different person by varying the names of the characters within the scenarios (e.g., Frank was bad and Frank experienced a bad outcome vs. Geoff was bad and Mark experienced a bad outcome). Participants were randomly assigned to one of two sets of 8 scenarios ( 2 scenarios per each condition). The scenarios were the same across sets except that the names of the characters changed. Participants responded to the scenarios in a random order.

\section{Results}


Spatial distances between the behaviors and outcomes were fit with a linear mixed effects model using the Ime4 package (Bates, Maechler, Bolker, \& Walker, 2015, version 1.121) in R (R Core Team, 2019, version 3.6.0). The models included fixed effects for Person (different vs. same, coded -0.5 and +0.5 ), Congruency (incongruent vs. congruent, coded 0.5 and +0.5 ) and the Person $X$ Congruency interaction. We included random intercepts for participants and scenarios, and random slopes by participants for the effects of Person, Congruency and the Person X Congruency interaction (random effects were uncorrelated due to issues with model convergence). We used Satterthwaite approximations to calculate $p$-values using the ImerTest package (Kuznetsova, Brockhoff, \& Christensen, 2016, version 3.1-0) and we report $95 \%$ percentile bootstrap confidence intervals (2,000 resamples).

As shown in Table 1, for both experiments there were statistically significant main effects of Person and Congruency. More importantly, there were also statistically significant Person X Congruency interactions (see Figure 2).

Table 1. Effects of Moral Congruency, Person who Experienced the Outcome, and Moral Congruency $X$ Person Interaction on Spatial Distances for Experiments 1 and 2.

\begin{tabular}{|c|c|c|c|c|}
\hline Predictor & $b$ (se) & $t(\mathrm{df})$ & $p$-value & $95 \% \mathrm{Cl}$ \\
\hline \multicolumn{5}{|l|}{ Experiment 1a } \\
\hline Person & $\begin{array}{l}-1.57 \\
(0.22)\end{array}$ & $\begin{array}{l}-7.06 \\
(27.39)\end{array}$ & $<.001$ & {$[-1.98,-1.13]$} \\
\hline Congruency & $\begin{array}{l}-1.01 \\
(0.20)\end{array}$ & $\begin{array}{l}-5.01 \\
(20.22)\end{array}$ & $<.001$ & {$[-1.40,-0.61]$} \\
\hline Person X Congruency & $\begin{array}{l}-1.16 \\
(0.35)\end{array}$ & $\begin{array}{c}-3.31 \\
(12.29)\end{array}$ & .006 & {$[-1.82,-0.48]$} \\
\hline $\begin{array}{l}\text { Congruency } \\
\text { (same persons) }\end{array}$ & $\begin{array}{l}-1.59 \\
(0.26)\end{array}$ & $\begin{array}{c}-6.15 \\
(15.20)\end{array}$ & $<.001$ & {$[-2.10,-1.06]$} \\
\hline $\begin{array}{l}\text { Congruency } \\
\text { (different persons) }\end{array}$ & $\begin{array}{l}-0.43 \\
(0.25)\end{array}$ & $\begin{array}{l}-1.72 \\
(12.68)\end{array}$ & .11 & {$[-0.88,0.05]$} \\
\hline
\end{tabular}

\section{Experiment 1b}

$\begin{array}{lcccc}\text { Person } & -16.16 & -6.25 & <.001 & {[-20.85,-10.95]} \\ & (2.59) & (15.25) & & \\ \text { Congruency } & -15.16 & -5.46 & <.001 & {[-20.57,-9.55]} \\ & (2.78) & (18.72) & & \\ \text { Person X Congruency } & -10.84 & -2.36 & .038 & {[-19.75,-1.73]}\end{array}$




$\begin{array}{lcccc}\text { Congruency } & -20.59 & -5.81 & <.001 & {[-27.39,-13.34]} \\ \text { (same persons) } & (3.55) & (15.25) & & \\ \text { Congruency } & -9.75 & -2.92 & .014 & {[-16.05,-3.42]} \\ \text { (different persons) } & (3.34) & (10.87) & .014 & \end{array}$

Note. Simple effects of Congruency within the Person conditions by experiment are shown in italics.

Follow-up analyses by refitting the models using dummy coding revealed that participants moved the outcomes closer to the immoral actions when they were congruent (vs. incongruent) more strongly when the outcomes occurred to the same persons than to different persons (see Table 1).
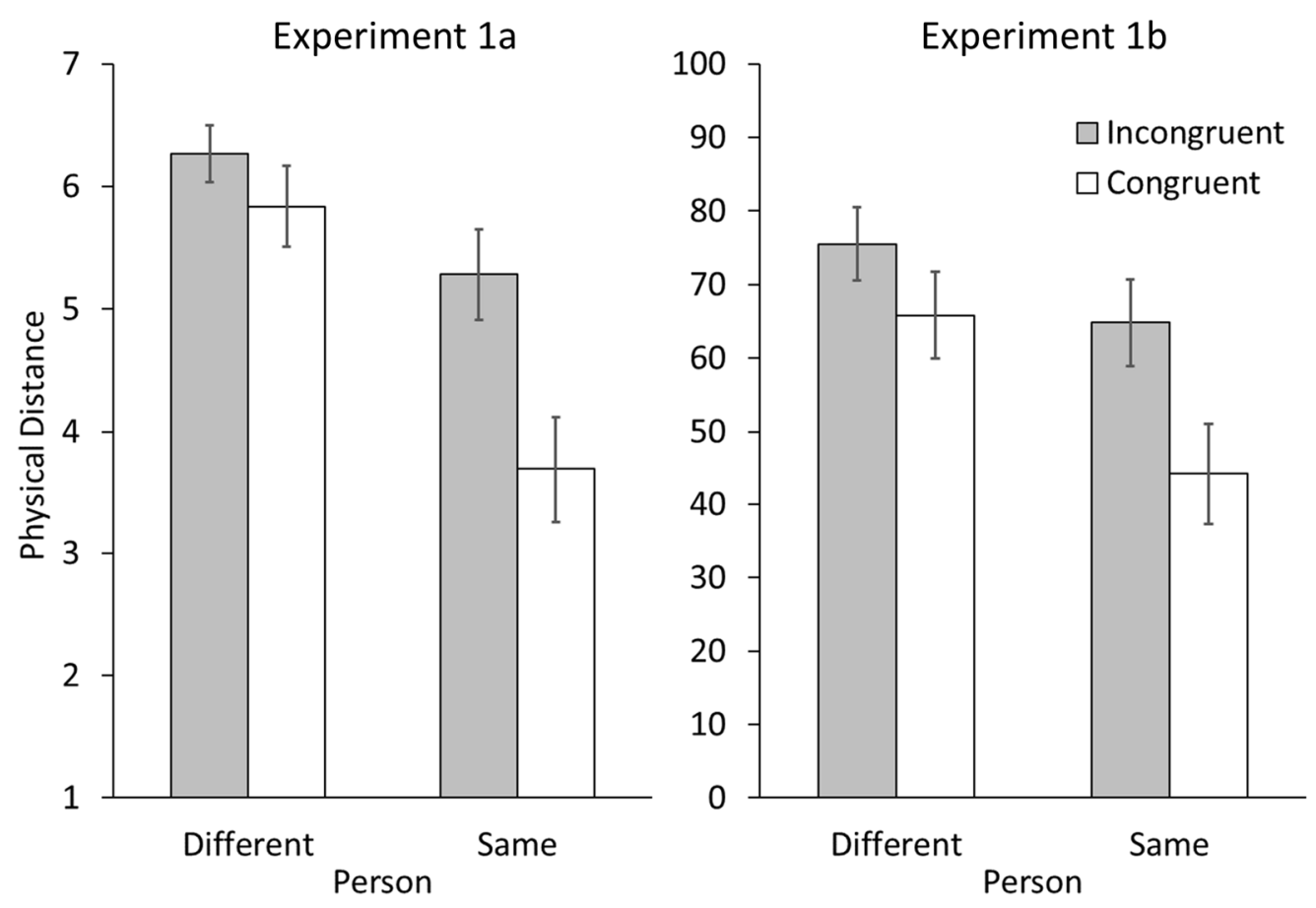

Figure 2. The effect of moral congruency on the distance between the immoral behaviors and outcomes as a function of whether the outcomes occurred to the same vs. different persons (Experiments $1 \mathrm{a}$ and $1 \mathrm{~b}$ ). Error bars show $95 \%$ confidence intervals of the mean.

\section{Experiment 2}

Experiments $1 \mathrm{a}$ and $1 \mathrm{~b}$ found that participants placed the events closer together in space when they were morally congruent (vs. incongruent), and they did so more strongly when the outcomes occurred to the same (vs. different) persons. These interaction patterns are important because they help discount perceived similarity as an explanation for the spatial binding of immoral actions and negative outcomes. If perceived similarity alone was driving the patterns we observed, then we would have expected only main effects (i.e., the effects 
of the manipulations would be additive). Indeed, in a supplementary study, we found that when asked to rate the similarity of the scenarios used in these experiments, both the Congruency and Person factors influenced similarity, but no interaction between the two was observed (see supplementary materials).

Although these experiments discount perceived similarity as an explanation, they do not provide evidence for our assumption that the spatial binding of immoral actions and negative outcomes are associated with perceived deservingness. To this end, in Experiment 2 we asked participants to rate the degree to which they believed a target person deserved a good or bad outcome along with completing the spatial binding task from Experiment 1a. We predicted that perceived deservingness of the outcome would mediate the effect of congruency on the spatial positioning of the action and outcome.

\section{Method}

Participants. Participants were recruited to complete an online survey $(N=207,87$ males, 1 unreported; $\left.M_{\text {age }}=36.59, S D_{\text {age }}=12.65\right)$. An additional 16 participants were excluded due to duplicate IP addresses $(n=5)$ or failing an attention check item $(n=11)$.

Materials and procedure. Experiment 2 was like Experiment 1a except that participants moved sentences for only one of two scenarios and rated the degree to which they believed the target deserved the outcome. The sentence associated with the target's immoral action was fixed across conditions: "Frank forcefully raped a co-worker at a company retreat". Varied between-subjects, the sentence associated with the target's outcome involved the performance of his financial investments. In the congruent outcome condition, participants read, "Frank's stocks and shares plummeted, netting him a massive financial loss", whereas participants in the incongruent outcome condition read, "Frank's stocks and shares skyrocketed, netting him a massive financial gain". Thus, both the target's immoral action and the initial event that generated the outcome were identical between conditions-only the value of the outcome varied between conditions.

Participants moved the sentences as near or far apart from each other as they wanted (see top section of Figure 1). Next, participants rated the degree to which they believed Frank deserved the outcome ( 1 = not at all deserving to $7=$ very deserving; $\mathrm{cf}$. Harvey et al., 2017). Finally, participants completed a multiple-choice attention check item (“What happened to Frank's financial investments?”).

\section{Results and discussion}

Participants rated the target as more deserving of his outcome when his financial investments plummeted $(M=6.26, S D=1.24)$ than when they skyrocketed $(M=1.67, S D=$ 
1.24), $t(198.27)=24.14, p<.001, d=3.36$ (degrees of freedom were Welch corrected). Conceptually replicating Experiment 1a, participants who learned that the target's stocks and shares plummeted $(M=3.61, S D=2.37)$ moved the sentences closer together than did participants who learned that they skyrocketed $(M=5.52, S D=2.18), t(203.27)=-6.03, p<$ $.001, d=-0.84$.

Perceived deservingness and how close together participants positioned the events were significantly correlated $(r=-.44, p<.001)$. We tested the indirect effect of moral congruency on distance between events through perceived deservingness using the quasiBayesian Monte Carlo method (5,000 simulations) with the 'mediation' package (Tingley, Yamamoto, Hirose, Keele, \& Imai, 2014; see Imai, Keele, \& Tingley, 2010) in R. As shown in Figure 3, perceived deservingness significantly mediated the effect of moral congruency on spatial distance between events (indirect effect $=-1.79,95 \%$ Monte Carlo confidence interval: $-2.84,-0.75 ; p=.001)$. These findings support the hypothesis that participants' spatial binding of bad outcomes to bad people are associated with perceived deservingness. This occurred when the same process that generated the outcome (financial investing) led to either a fortuitous good or bad outcome for the immoral target, and they replicate our findings from Experiments $1 \mathrm{a}$ and $1 \mathrm{~b}$ using a between-subjects design (which is not susceptible to carry-over or expectation effects).

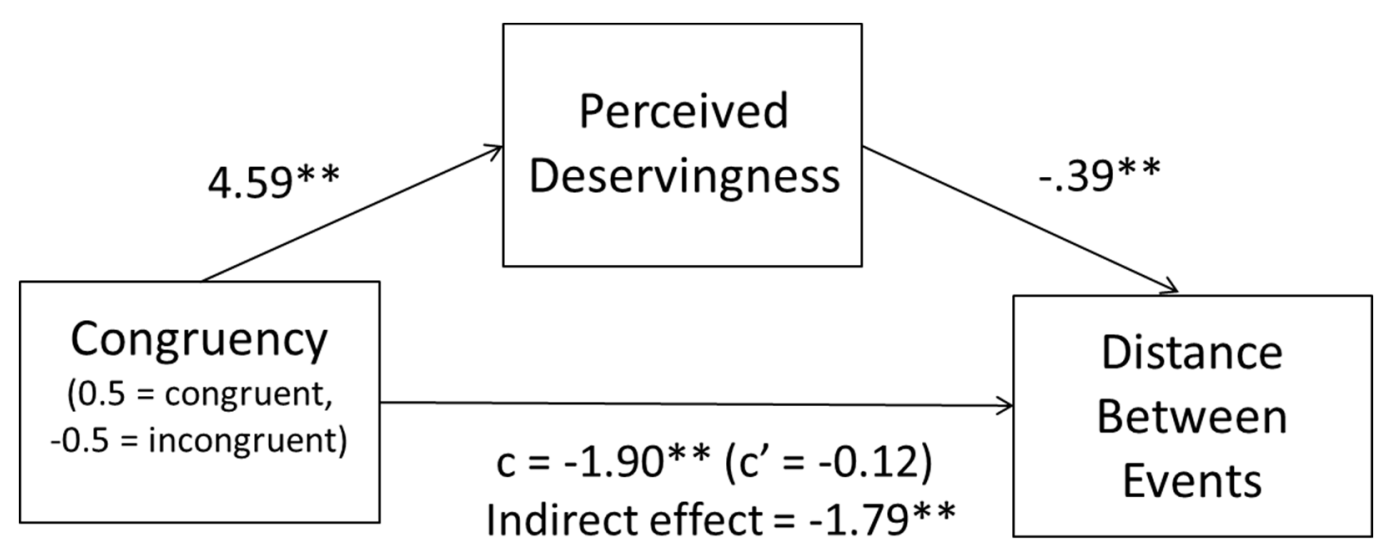

Figure 3. Mediation model for Experiment 2. Values depict unstandardized regression coefficients. ${ }^{* *} p<.01$.

\section{Experiment 3}

Experiment 3 had two key aims: (1) to generalize our previous findings using a different, more dynamic spatial positioning task and (2) to test the relative importance of perceived deservingness and perceived similarity in the spatial binding of actions and outcomes. 
One limitation of Experiment 2 is that spatial positioning and perceived deservingness were measured simultaneously. It is possible that participants' ratings of deservingness were contaminated by their spatial positioning of the action and outcomes, such that participants might have simply inferred the target's deservingness from how physically near or far apart they happened to position the outcome from the action. To address this issue, in Experiment 3 participants completed a spatial binding task or provided ratings of deservingness; scenarios formed the basic unit of analysis. Specifically, one sample of participants completed only a spatial positioning task which included several scenarios varying in terms of the moral congruency of the outcomes experienced by the targets. The task we used in Experiment 3 was more active and dynamic than the tasks in Experiments 1a and $1 \mathrm{~b}$, such that participants were asked to keep a "chasing" ball representing a target's immoral behaviour as close or far as they wanted from a ball representing a good or bad outcome. A different sample of participants rated the deservingness of the outcomes for the targets across the same scenarios used in the spatial binding task (Part 1a), and we explored the association between deservingness and spatial binding of outcomes to immoral actions at the level of the scenarios.

Using scenarios as the unit of analysis allowed us to address two further questions: First, we examined whether deservingness per se is associated with the spatial binding of bad outcomes to immoral actions or whether perceived similarity between actions and outcomes confounds this association. We asked a third sample of participants to rate how similar they believed the actions (e.g., drowning a puppy) were to the outcomes (e.g., a fractured leg) across the same scenarios used for the "chasing" task (Part 1b). On the one hand, if perceived deservingness correlates with the spatial binding of outcomes to actions simply because of its association with perceived similarity, then we would not expect deservingness to uniquely predict spatial binding over and above the contributions of perceived similarity. On the other hand, finding that perceived deservingness uniquely predicts spatial binding over and above perceived similarity would suggest that its association is not simply due to how conceptually similar people perceive the events under question. Second, to provide convergent validity for our spatial binding task as reflecting causal attributions, we asked another sample of participants to rate the degree to which they believed the outcomes were a result of the targets' immoral behaviour (Part 1c; cf. Callan et al., 2006).

\section{Method}


Participants. We recruited 43 (29 males; $\left.M_{\text {age }}=32.98, S D_{\text {age }}=10.02\right), 43$ (20 males, 1 non-binary; $\left.M_{\text {age }}=33.37, S D_{\text {age }}=9.90\right)$ and 43 (27 males, 1 non-binary; $M_{\text {age }}=33.37, S D_{\text {age }}=$ 9.90) participants for Parts 1a, 1b, and 1c, respectively. For Part 2 ("chasing" binding task), we recruited 75 participants (42 males, 1 preferred not to say; $M_{\text {age }}=33.40, S D_{\text {age }}=9.15$ ) ${ }^{1}$

Materials and procedures.

Part 1a: perceived deservingness. For Part 1a, participants responded to 64 scenarios describing someone engaging in an immoral action (e.g., "Joe robbed a store at gunpoint") and experiencing either a good or bad outcome (e.g., "Joe was in a freak car accident"). We used the same 8 immoral behaviors and 8 outcomes ( 4 good, 4 bad); the outcomes were cycled through the 8 immoral behaviors to create 64 individual scenarios. For each scenario, participants rated the extent to which they believed the target person deserved the outcome that they experienced using a 1 (not at all deserving) to 7 (very deserving) scale, which was shown directly below the two sentences describing the action and outcome.

Part 1b: perceived similarity. Part $1 \mathrm{~b}$ was the same as Part 1a except that participants responded to pairings of the actions ("Robbing a store at gunpoint") and outcomes ("A freak car accident") without mention of who performed the actions or experienced the outcomes. This allowed us to gauge perceived similarity of actions and outcomes at the conceptual level and in a way that did not imply perceived deservingness of the outcome (or lack thereof) for any particular target. For each scenario, participants rated the extent to which they believed that the events were similar using a 1 (not at all similar) to 7 (very similar) scale (cf. Casasanto, 2008).

Part 1c: perceived causality. For each of the 64 scenarios used in Parts $1 \mathrm{a}$ and $1 \mathrm{~b}$, in Part 1c participants rated the extent to which they believed the outcome was a result of the target's prior immoral behaviour (e.g., "To what extent do you believe that James's stocks and shares skyrocketing was a result of him cheating on his wife?") using 1 (not at all) to 7 (a great deal) scale.

Part 2: spatial positioning. Participants controlled the movements of a circle representing the outcome (a white circle) while it was "chased" by a circle representing the initial immoral behavior (a red circle; see Figure 1). Both circles started each trial in the center of the screen on the vertical plane, and at $30 \%$ and $70 \%$ of the screen on the horizontal plane. Participants pressed one of the arrow keys to move the white circle. The

\footnotetext{
${ }^{1}$ We collected data from 7 additional participants to address imbalances in the number of participants completing the different sets of stimuli (see below).
} 
circle moved one-step ( $4 \%$ of the screen) every $300 \mathrm{~ms}$ provided the participant had pressed during that time. The red circle moved one-step ( $2 \%$ of the screen size) in the direction of the white circle every $300 \mathrm{~ms}$. Each trial lasted 15 seconds, and participants could move the white circle away from or towards the red circle to keep them as close together or as far apart as they wanted. We calculated the distance between the two circles on each sample (every $300 \mathrm{~ms}$ ) by taking the mean of their vertical distance and horizontal distance, both calculated as a percentage of the maximal distance on each axis. We calculated the average distance across the course of the trial by taking the mean distance over each of the 50 samples.

Following a practice trial with neutral sentences (per Experiment 1a), participants responded to four congruent (e.g., "Joe robbed a store at gun point" and "Joe's apartment was destroyed by fire") and four incongruent (e.g., "Tim kidnapped a child" and "Tim received a major pay rise at work") scenarios. Participants were randomly assigned to 1 of 8 sets of 8 scenarios. The immoral actions were the same across sets; the differences between the sets was that 8 outcomes sentences ( 4 good, 4 bad) were cycled through the sets such that each outcome was paired with each "behaviour" once across the sets. The scenarios were presented in a random order across participants.

\section{Results}

Effects of congruency. Data from each part of Experiment 3 were separately submitted to a linear mixed effects model which included a fixed effect for Congruency (incongruent vs. congruent, coded -0.5 and +0.5 ), random intercepts for participants and scenarios, and random slopes by participants for the effect of Congruency. As shown in Table 1, analyses revealed a statistically significant effect of Congruency for all measures. Importantly, participants actively kept the outcome circles closer to the "chasing" immoral action circles more when they were congruent (vs. incongruent).

Table 2. Effects of congruency on perceived deservingness, perceived similarity, causal judgments, and physical distance during the "chasing" spatial binding task (Experiment 3).

\begin{tabular}{lcccccc}
\hline & Congruent & \multicolumn{2}{l}{ Incongruent } & & & \\
\cline { 2 - 6 } Variable & $M(S D)$ & $M(S D)$ & $b(\mathrm{se})$ & $t(\mathrm{df})$ & $p$-value & $95 \% \mathrm{Cl}$ \\
\hline Perceived & 5.81 & 1.48 & 4.33 & 15.62 & $<.001$ & {$[3.79,4.90]$} \\
deservingness & $(1.21)$ & $(0.95)$ & $(0.28)$ & $(42.28)$ & & \\
Perceived & 2.80 & 2.56 & 0.24 & 2.55 & & \\
similarity & $(1.78)$ & $(1.78)$ & $(0.10)$ & $(49.11)$ & .014 & {$[0.07,0.43]$}
\end{tabular}




\begin{tabular}{lcccccc} 
Causal & 2.86 & 2.03 & 0.83 & 4.41 & $<.001$ & {$[0.48,1.21]$} \\
judgments & $(1.62)$ & $(1.55)$ & $(0.19)$ & $(52.68)$ & & \\
Physical & 16.42 & 22.41 & -6.00 & -6.47 & & \\
distance & $(7.65)$ & $(6.04)$ & $(0.93)$ & $(69.71)$ & $<.001$ & {$[-7.78,-4.28]$} \\
\hline
\end{tabular}

Predicting spatial distance from deservingness and similarity. Ratings of the predictors were highly reliable across scenarios (all $\alpha>$.94). As shown in Table 3, with scenario as the basic unit of analysis, perceived similarity and deservingness correlated significantly with each other, and they each correlated significantly with the distance participants actively kept the events away from each other. Providing convergent validity for the spatial binding measure, ratings of perceived causality correlated significantly (though not perfectly) with physical distance. ${ }^{2}$ That is, the more participants rated the events as causally related, the closer together they kept them during the "chasing" task.

Table 3. Intercorrelations among scenario-level variables (Experiment 3).

\begin{tabular}{|c|c|c|c|c|c|}
\hline Variables & 1. & 2. & 3. & 4. & 5. \\
\hline $\begin{array}{l}\text { 1. Condition } \\
(0.5=\text { congruent },- \\
0.5=\text { incongruent })\end{array}$ & -- & & & & \\
\hline $\begin{array}{l}\text { 2. Perceived } \\
\text { Deservingness }\end{array}$ & $.998^{*}$ & -- & & & \\
\hline $\begin{array}{l}\text { 3. Perceived } \\
\text { Similarity }\end{array}$ & $.60 *$ & $.59 *$ & -- & & \\
\hline 4. Causal judgments & $.81 *$ & $.82 *$ & $.52 *$ & -- & \\
\hline 5. Physical distance & $-.75 *$ & $-.75^{*}$ & $-.44 *$ & $-.71 *$ & -- \\
\hline
\end{tabular}

Note. $N=64 .{ }^{*} p<.001$.

We tested the unique contributions of deservingness and similarity by fitting the distances participants kept the events away from each other with a linear mixed effects model which included fixed effects for perceived similarity and perceived deservingness, random intercepts for participants and scenarios, and random slopes by participants for the effects of perceived deservingness and similarity. Analyses revealed that perceived

\footnotetext{
${ }^{2}$ In an additional validation study, 85 participants completed the "same person" scenarios from the task used in Experiment 1a and rated the degree to which they believed the outcome was the result of the target's prior behavior. Consistent with these scenario-level findings, the closer participants moved together the actions and outcomes overall, the more they believed the outcomes were a result of the target's prior actions, $r=-0.54, p<.001$ (see supplementary materials).
} 
deservingness, $b=-1.36$, se $=0.21, \mathrm{Cl}=[-1.76,-0.94] ; t(83.81)=-6.48, p<.001$, but not perceived similarity, $b=0.43$, se $=1.76, \mathrm{Cl}=[-3.93,2.93] ; t(51.35)=-0.24, p=0.81$, uniquely predicted spatial distances during the chasing task. These findings suggest that the role of perceived deservingness in the spatial binding of outcomes to immoral actions is not simply due to its covariation with the perceived similarity of the actions and outcomes themselves.

\section{General Discussion}

We found that participants positioned representations of others' immoral actions and fortuitous outcomes spatially closer when the outcomes were perceived as deserved. Negative outcomes were positioned closer to previous immoral behaviors than were positive outcomes (Experiments 1a to 2), and when controlling the movement of a positive or negative outcome that was being "chased" on screen by a bad person, participants kept positive outcomes further away from bad people (Experiment 3). This suggests that not only did participants position bad outcomes and bad people closer together, they actively tried to keep good outcomes away from bad people. ${ }^{3}$

Although previous research has documented that people, at times, casually attribute others' random bad outcomes to their prior misdeeds, none of this work specifically tested perceived similarity of the events as a potential alternative explanation. We systematically addressed this in several ways. First, we found that this spatial positioning bias is significantly greater when the two scenarios describe the same protagonist. Although a cheating spouse and a car crash victim might be positioned close together because they are affectively similar events, this spatial bias is most prominent when the negative event has befallen the person (vs. not the person) committing the immoral act. In Experiment 2, we extend this finding to show that this spatial bias is explained by the perceived deservingness of the outcome. Finally, in Experiment 3 we found that deservingness predicts spatial positioning over and above any association with perceived similarity. Taken together, these findings suggest that immanent justice reasoning, as measured by spatial proximity, is underpinned more by perceived deservingness than by perceived similarity of the events.

The spatial positioning tasks we developed here may be less susceptible to selfreport bias than explicit causal ratings used in most previous research in this field. In such studies, although agreement is greater for morally congruent outcomes (compared to

\footnotetext{
${ }^{3}$ Across our studies we used scenarios involving immoral targets only. From just-world theory, however, we should observe the same effects of moral congruency on spatial positioning using situations involving moral/good targets (cf. Callan et al., 2006; Harvey et al., 2017; White et al., 2019). To this end, in a pre-registered conceptual replication, we found that participants moved outcomes closer to moral actions when the outcomes were good (vs. bad; see supplementary materials).
} 
morally incongruent outcomes), at least Western participants rarely highly agree with immanent justice explanations for chance outcomes (e.g. Callan et al., 2006), presumably because doing so flies in the face of conventional rationality and meta-physical realism. Indeed, raping a co-worker, for example, does not ipso facto cause fluctuations in the stock markets, and participants' explicit causal judgments tend to accord with this lack of physical causality. Because the tasks we developed gauge immanent justice reasoning indirectly, they may be more suitable in research contexts or among individuals where the motivation to suppress the expression of immanent justice explanations is more important. Future research in this area would therefore benefit from investigating individual differences and situations in which the spatial positioning of moral actions and outcomes might converge with other constructs of theoretical importance to immanent justice reasoning, such as religiosity, magical ideation, socialization of justice beliefs, and the importance of a justworld to people (see Callan et al., 2014). Moreover, it is important to highlight that these studies were conducted using Western samples which limits the generalizability of our findings. Although immanent justice reasoning has been observed in Asian cultural contexts (e.g., Murayama \& Miura, 2016; White, Norenzayan, \& Schaller, 2019), the extent of any cross-cultural differences or similarities in immanent justice reasoning by spatial proximity is yet to be investigated.

\section{Conclusions}

The current work suggests that when making sense of people's immoral actions and their ensuing outcomes, people spatially bind together morally congruent events to fit the idea that people get what they deserve. This work therefore contributes to theory and research on the conceptual metaphorical link between physical closeness and causal influence (Lakoff \& Johnson, 1980) and provides new insights into the role that perceived deservingness, over and above perceived similarity of actions and outcomes, plays in immanent justice reasoning. 


\section{References}

Bates, D., Maechler, M., Bolker, B., \& Walker, S. (2015). Fitting Linear Mixed-Effects Models Using Ime4. Journal of Statistical Software, 67(1), 1-48.

Breaux, B. O., \& Feist, M. I. (2008). The color of similarity. In B. C. Love, K. McRae, \& V. M. Sloutsky (Eds.), Proceedings of the Annual Meeting of the Cognitive Science Society, 30 (30) (pp. 253-258).

Buehner, M. J., \& Humphreys, G. R. (2010). Causal contraction: spatial binding in the perception of collision events. Psychological Science, 21, 44-48.

Boot, I., \& Pecher, D. (2010). Similarity is closeness: Metaphorical mapping in a conceptual task. The Quarterly Journal of Experimental Psychology, 63(5), 942-954.

Callan, M. J., Ellard, J. H., \& Nicol, J. E. (2006). The belief in a just world and immanent justice reasoning in adults. Personality and Social Psychology Bulletin, 32, 1646-1658.

Callan, M. J., Harvey, A. J., Dawtry, R. J., \& Sutton, R. M. (2013). Through the looking glass: Focusing on long-term goals increases immanent justice reasoning. British Journal of Social Psychology, 52(2), 377-385.

Callan, M. J., Sutton, R. M., Harvey, A. J., \& Dawtry, R. J. (2014). Immanent justice reasoning: Theory, research, and current directions. In J. M. Olson, \& M. P. Zanna (Eds.), Advances in Experimental Social Psychology, Vol. 49. (pp. 105-161). London: Academic Press.

Casasanto, D. (2008). Similarity and proximity: When does close in space mean close in mind? Memory \& Cognition, 36, 1047-1056.

Einhorn, H. J., \& Hogarth, R. M. (1986). Judging probable cause. Psychological Bulletin, 99(1), 3-19.

Faro, D., Leclerc, F., \& Hastie, R. (2005). Perceived causality as a cue to temporal distance. Psychological Science, 16(9), 673-677.

Goldstone, R. (1994). An efficient method for obtaining similarity data. Behavior Research Methods, Instruments, \& Computers, 26, 381-386.

Harvey, A. J., \& Callan, M. J. (2014). Getting "just deserts" or seeing the "silver lining": The relation between judgments of immanent and ultimate justice. PloS one, 9(7), e101803.

Harvey, A. J., Callan, M. J., Sutton, R. M., Foulsham, T., \& Matthews, W. J. (2017). Selective exposure to deserved outcomes. Journal of Experimental Social Psychology, 69, 33-43. 
Hout, M. C., Goldinger, S. D., \& Ferguson, R. W. (2013). The versatility of SpAM: A fast, efficient, spatial method of data collection for multidimensional scaling. Journal of Experimental Psychology: General, 142(1), 256-281.

Imai, K., Keele, L., \& Tingley, D. (2010). A general approach to causal mediation analysis. Psychological Methods, 15(4), 309.

Kuznetsova, K., Brockhoff, P. B., \& Christensen, R. H. B. (2016). ImerTest: Tests in Linear Mixed Effects Models. R package version 2.0-36. http://CRAN.R-project.org/ package=ImerTest

Lakoff, G., \& Johnson, M. (1980). Metaphors we live by. Chicago: University of Chicago Press. Landau, M. J., Meier, B. P., \& Keefer, L. A. (2010). A metaphor-enriched social cognition. Psychological Bulletin, 136(6), 1045-1067.

Lerner, M. J. (1980). The belief in a just world: A fundamental delusion. New York: Plenum. Michotte, A.E. (1963). The perception of causality (Miles, T.R., Trans.). London: Methuen \& Co. (Original work published 1946).

Murayama, A., \& Miura, A. (2016). Two types of justice reasoning about good fortune and misfortune: A replication and beyond. Social Justice Research, 29(3), 331-344.

Piaget, J. (1932/1965). The moral judgment of the child. London, England: Kegan, Paul, Trench, Trubner.

R Core Team (2019). R: A language and environment for statistical computing. R Foundation for Statistical Computing, Vienna, Austria. URL https://www.R-project.org/.

Rips, L. J. (2011). Causation from perception. Perspectives on Psychological Science, 6(1), 7797.

Tingley, D., Yamamoto, T., Hirose, K., Keele, L., \& Imai, K. (2014). Mediation: R package for causal mediation analysis. Journal of Statistical Software, 59(5), 1-38.

Thibodeau, P. H., Matlock, T., \& Flusberg, S. J. (2019). The role of metaphor in communication and thought. Language and Linguistics Compass, 13(5), e12327.

White, C. J., Norenzayan, A., \& Schaller, M. (2019). The content and correlates of belief in Karma across cultures. Personality and Social Psychology Bulletin, 45(8), 1184-1201.

White, P. A. (1988). Causal processing: Origins and development. Psychological Bulletin, 104(1), 36-52.

Winter, B., \& Matlock, T. (2013). Making judgments based on similarity and proximity. Metaphor and Symbol, 28(4), 219-232. 


\section{SUPPLEMENTARY MATERIALS FOR “IMMANENT JUSTICE BY SPATIAL PROXIMITY”}

\section{Supplementary Experiment A: Perceived Similarity}

In Supplementary Experiment A, we examined the potential interactive effect of congruency of the outcome (congruent vs. incongruent) and the person who experienced the outcome (same vs. different person) on perceived similarity of the events.

\section{Method}

Participants. Sixty-two participants $\left(63 \%\right.$ male: $\left.M_{\text {age }}=31.35, S D_{\text {age }}=6.80\right)$ completed a brief online survey about people's perceptions of similarity.

Materials and procedures. Supplementary Experiment A was the same as the main Experiment 1 except that instead of moving sentences in space, participants rated the extent to which they believed the events described in each of the 16 scenarios were similar ("How similar are these two events?"; 1 = not at all similar to 7 = very similar). There were 4 scenarios for each of the 4 conditions of the fully-within Person X Congruency design. Participants saw the behaviour (e.g., "James robbed a convenience store at gunpoint") and outcome (e.g., "Charlie won $\$ 100,000$ from a scratch-and-win lottery ticket") sentences listed on separate lines, with the rating scale displayed directly below the sentences. The scenarios were presented in a random order. Like our main studies, the materials and data for all of the supplementary studies are available at osf.io/fb7g5/.

\section{Results}

Participants' ratings of perceived similarity were analysed using mixed effects regression as per Experiment 1. Analyses revealed main effects for Person, $b=0.35$, se $=0.10,95 \% \mathrm{Cl}=$ $[0.16,0.54] ; t(28.82)=3.53, p=.001$, and Congruency, $b=0.16$, se $=0.08,95 \% \mathrm{Cl}=[0.01$, $0.32] ; t(15.23)=2.00, p=.06$. More importantly, as shown in Figure $\mathrm{S} 1$, there was no significant Person $X$ Congruency interaction, $b=0.01$, se $=0.15,95 \% \mathrm{Cl}=[-0.29,0.32]$; $t(12.38)=0.08, p=.94$. If perceived similarity was accounting for the spatial binding effects we observed in Experiments 1a and 1b, then we would have expected to show a similar interaction pattern for similarity judgments, whereby the effect of Congruency on perceived similarity would have been stronger when the outcome occurred to the same (vs. different) person, perhaps because participants were giving more weight to the co-occurrences of common features in the scenarios when determining similarity. Instead, these supplementary findings suggest that perceptions of similarity follow an additive rather than an interactive pattern for this design. 


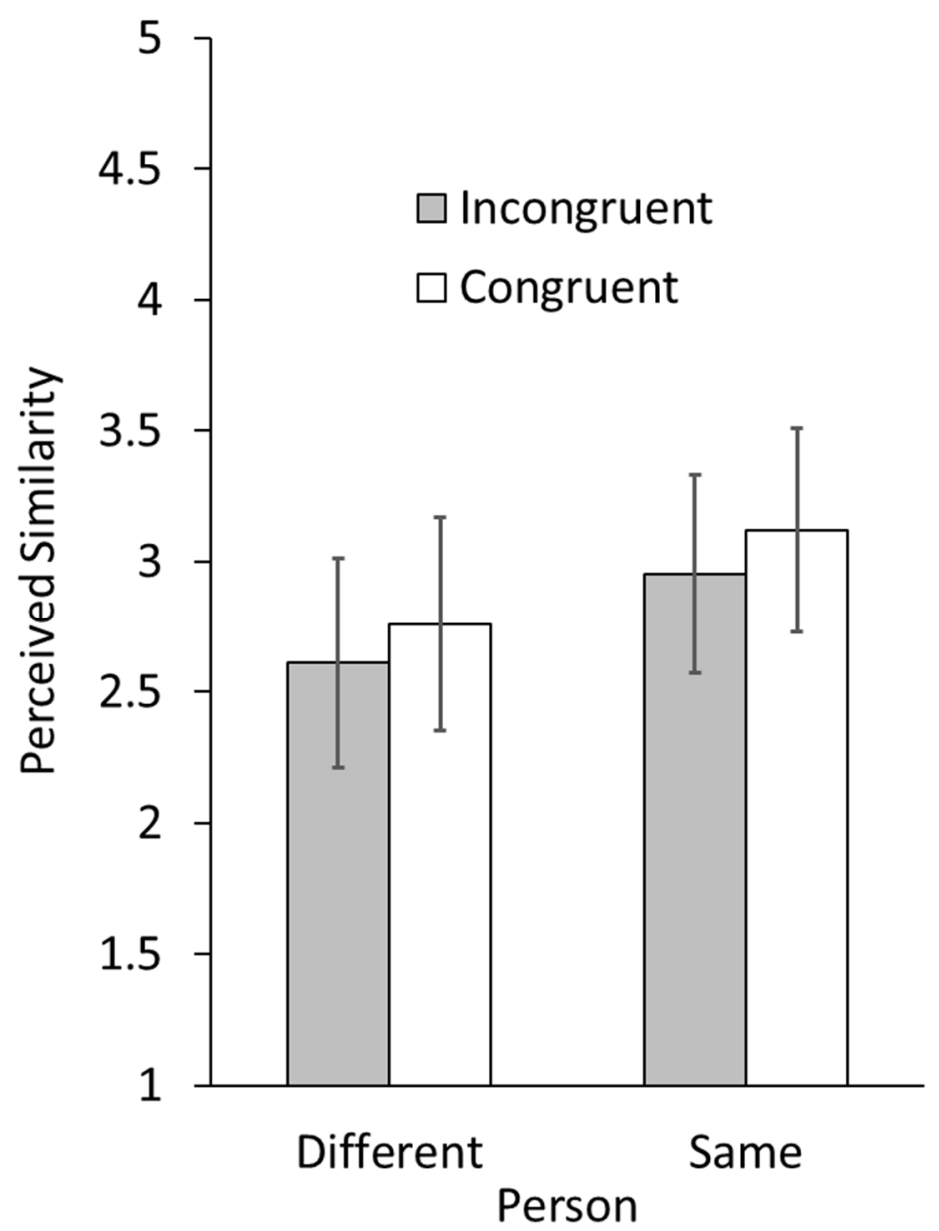

Figure S1. The effect of moral congruency on perceived similarity of the events as a function of whether the outcomes occurred to the same vs. different persons (Supplementary Experiment A). Error bars show $95 \%$ confidence intervals of the mean.

\section{Supplementary Experiment B: Perceived Causality and Spatial Closeness}

In Supplementary Experiment B, we sought evidence for the convergent validity of the spatial arrangement task used in Experiment 1a. We did so by examining the association between ratings of causality and how close together participants positioned the actions and outcomes during the task.

Method

Participants. Eighty-five participants ( $53 \%$ male: $\left.M_{\text {age }}=35.36, S D_{\text {age }}=11.86\right)$ completed a brief online survey about people's perceptions of physical closeness between events.

Materials and procedures. Supplementary Experiment B was similar to the main Experiment 1a except that along with moving action and outcome-related sentences in space, participants rated the extent to which they believed the outcome was a result of the 
target's prior immoral behaviour using 1 (not at all) to 7 (a great deal) scale (cf. the main Experiment 3).

For both the causal judgments and the spatial positioning task, participants responded to the "same person" condition only (cf. the main Experiment 3). Half of the 8 total trials/ratings were congruent and half were incongruent. Participants completed either the spatial binding task first or the causal judgments first (randomly determined), and the order of the scenarios/trials within blocks was randomized.

\section{Results}

Participants' spatial positioning of the sentences and ratings of causality were analysed using mixed effects regression as per Experiment 3. Replicating our Experiment 1a effect, participants moved the outcomes closer to the immoral actions when they were congruent than when they were incongruent, $b=-1.06$, se $=0.32,95 \% \mathrm{Cl}=[-1.67,-0.46] ; t(10.65)=-$ 3.34, $p=.007$. Replicating our main Experiment 3 (Part $C$ ) and previous research on immanent justice reasoning, participants believed the outcomes were a result of the target's prior conduct more when they were morally congruent than when they were incongruent, $b$ $=0.84$, se $=0.30,95 \% \mathrm{Cl}=[0.28,1.39] ; t(2.82)=2.82, p=.02$.

Providing convergent validity for the spatial binding measure as gauging causal reasoning, when averaged across scenarios/trials, ratings of causality correlated significantly with physical distance, $r(83)=-.54, p<.001$, such that the more participants believed the outcomes were caused by the targets' prior behaviours, the closer together they positioned the actions and outcomes. Fitting the distances participants positioned the actions and outcomes during the spatial binding task with a linear mixed effects model including a fixed effect for perceived causality, random intercepts for participants and scenarios, and random slopes by participants for the effect of perceived causality also revealed a significant relationship, $b=-0.68$, se $=0.05, \mathrm{Cl}=[-0.78,-0.58] ; t(61.31)=-13.43, p<.001$.

\section{Supplementary Experiment C: Spatial Binding of Good vs. Bad Outcomes to Moral Actions}

Across our main studies we used scenarios where the target persons always performed immoral actions. In Supplementary Experiment C, we conducted a pre-registered (https://aspredicted.org/xb3rr.pdf) conceptual replication of the effects of congruency on spatial binding of actions and good vs. bad outcomes using scenarios where the targets performed moral actions. Given that people tend to believe that good people deserve good outcomes more than they deserve bad outcomes (Callan et al., 2006; Harvey et al., 2017), using the spatial binding task from Experiment 1a, we expected that participants would position good (vs. bad) outcomes closer to sentences describing moral actions. 


\section{Method}

Participants. We requested 82 participants from Amazon's Mechanical Turk and over- recruited by six participants. The data from four participants were excluded due to duplicate IP addresses, leaving a final sample size of 84 participants ( $55 \%$ male: $M_{\text {age }}=33.00$, $\left.S D_{\text {age }}=9.24\right)$.

Materials and procedures. Supplementary Experiment C was similar to the "same person" condition in the main Experiment 1a except that participants moved sentences describing good and bad outcomes towards or away from sentences describing moral (i.e., good) behaviors (e.g., Frank saved a drowning child", "Ben mows his elderly neighbor's lawn"; See the top section of Figure 1 in the main text). We followed the design of our main Experiment 3 such that participants were randomly assigned to 1 of 8 sets of 8 scenarios (there were 64 scenarios in total). The moral actions were always the same across sets; the differences between the sets was that 8 outcomes sentences ( 4 good, 4 bad) were cycled through the sets such that each outcome was paired with each moral action once across the sets. The scenarios were presented in a random order across participants.

We therefore had two within-subjects conditions: congruent vs. incongruent outcomes. Like Experiment 1a, participants were asked to, for each trial, click and drag the sentences closer together or further apart from each other according to how physically close or far apart the two events felt to them. Each trial was scored from 1 (the sentences were placed directly next to each other) to 7 (the sentences were placed as far apart as possible).

\section{Results}

Following our pre-registered analysis plan, participants' spatial positioning of the sentences were submitted to a linear mixed effects model which included a fixed effect for Congruency (incongruent vs. congruent, coded -0.5 and +0.5 , respectively), random intercepts for participants and scenarios, and random slopes by participants for the effect of Congruency (random effects were correlated). Conceptually replicating the effects from our main studies involving immoral targets, participants moved the outcomes closer to the moral actions when they were congruent (i.e., good) than when they were incongruent (i.e., bad), $b=$ 1.07 , se $=0.25,95 \%$ bootstrapped $\mathrm{Cl}=[-1.57,-0.60] ; t(75.74)=-4.27, p<.001$. 